\title{
Recognizing Team Science Contributions in Academic Hiring, Promotion, and Tenure
}

\author{
${ }^{\circledR}$ Hollis Cline, ${ }^{1 \star}{ }^{\oplus}$ Lique Coolen, ${ }^{2 \star}$ Saskia de Vries, ${ }^{3 *}{ }^{\circledR}$ Steven Hyman, ${ }^{4 \star}{ }^{\circledR}$ Rosalind Segal,, $5 *$ and \\ ODswald Steward ${ }^{7 *}$ \\ ${ }^{1}$ Doris Neuroscience Center, Scripps Research Institute, La Jolla, CA 92037, ${ }^{2}$ Brain Health Research Institute, Kent State University, Kent, OH \\ 44242, ${ }^{3}$ Allen Institute for Brain Science, Seattle, WA 98109, ${ }^{4}$ Stanley Center for Psychiatric Research, Broad Institute, Cambridge, MA 02142, \\ ${ }^{5}$ Harvard Medical School, Boston, MA 02115, ${ }^{6}$ Dana-Farber Cancer Institute, Boston, MA 02215, and ${ }^{7}$ Reeve-Irvine Research Center, University of \\ California, Irvine, CA 92697
}

The vision of a scientist as a lone investigator reaching an epiphany is a widely cherished narrative. Consistent with this ideal, single-author papers were frequent 50 years ago, when the Society for Neuroscience started. However, the basic and translational questions and the public health challenges being addressed in current neuroscience research are increasingly interdisciplinary and multidimensional, and so the vast majority of significant studies require a team of investigators, working together collaboratively. This trend is evident in the increased number of authors per citation and the rapid expansion of collaborative grants (Fig. 1). Unfortunately, academic culture has not yet caught up with the direction of the science. Hiring, promotions, and peer review tend to credit the first and last authors, with little consideration that the work required an entire team. At the 2019 SfN annual meeting, there were two workshops addressing team science. One workshop highlighted the challenges in team science for trainees, whereas the other focused on ways in which academic leaders could change our procedures to address the disconnect between overly narrow attention to individual first and last authorship in hiring, promotion, and tenure versus the

\footnotetext{
Received May 20, 2020; revised June 20, 2020; accepted July 14, 2020 *Authors contributed equally and are listed alphabetically. The authors declare no competing financial interests.

This work was supported by National Institutes of Health Grant R25NS114922-01. We thank Michael Heintz, Sylvie Raver, and Maya Sapiurka of SfN for their help.

Correspondence should be addressed to Rosalind Segal at Rosalind_segal@dfci.harvard.edu.

https://doi.org/10.1523/JNEUROSCI.1139-20.2020

Copyright $(2020$ the authors
}
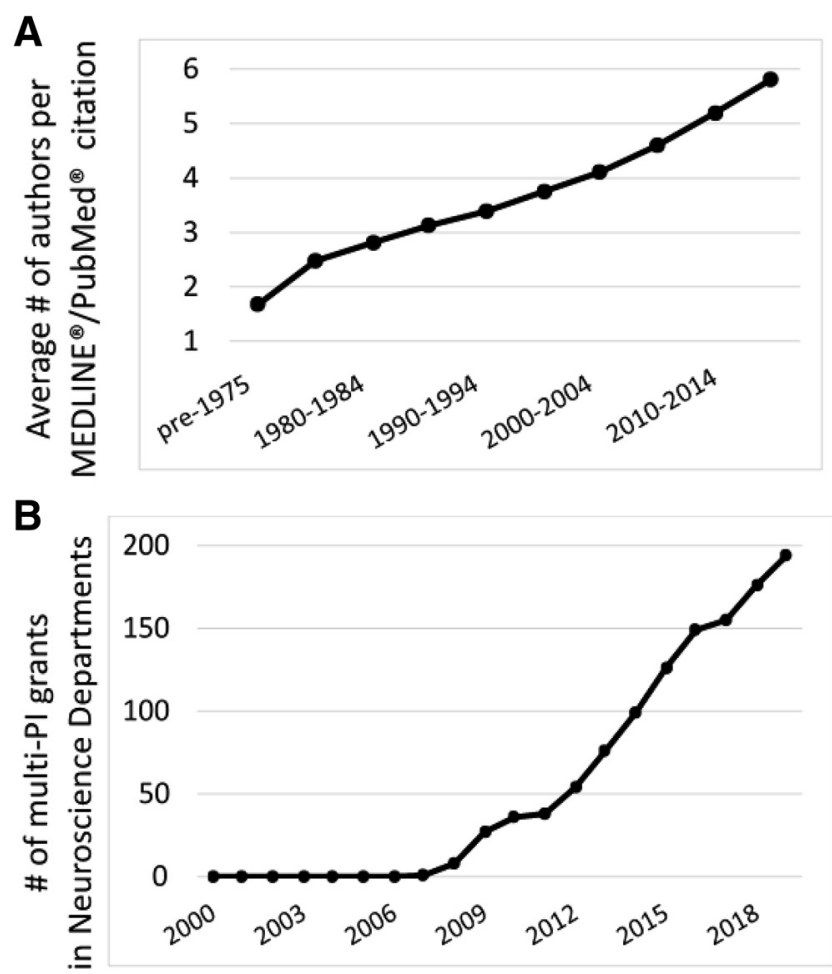

Figure 1. Team science over time. $\boldsymbol{A}$, The increase in authors per citation over time. Based on data from the National Library of Medicine. $\boldsymbol{B}$, The increase in number of multi-principal investigator (PI) grants from 2000 to 2018. Based on data from National Institutes of Health RePORTER.

collaborative nature of current research. This Commentary distills the ideas and recommendations brought forth by these workshops, to advocate for changes in academic recognition.

While some structural changes will be difficult to execute, there are innovations that could easily be conducted to increase the recognition of collaborative research. Among the simple solutions is a change in PubMed, Endnote, and other publication repositories. Currently, these do not include any recognition for co-first or cocorresponding authors. In this way, these databases fail to recognize even the current practices that acknowledge multiple contributors. We strongly advocate for this recognition of the current reality. The National Institutes of Health has already provided support for collaborations in the multi-principal investigator grants, and we recommend that other funding sources also allow more collaborative applications. Many publications now require listing the 


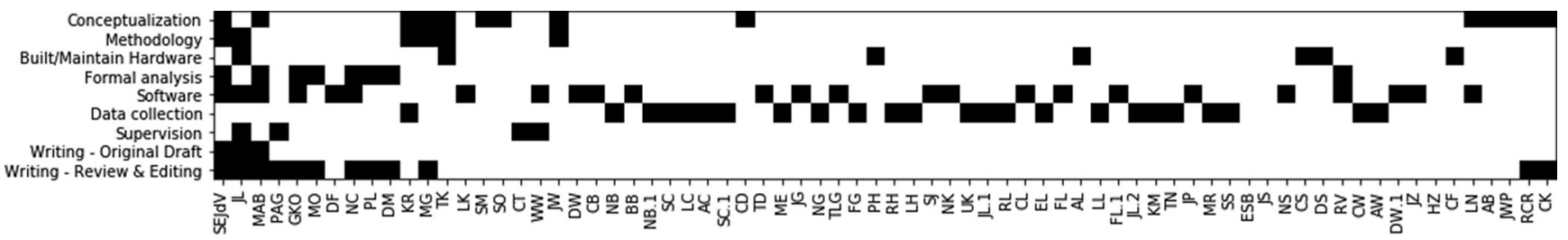

Figure 2. A proposed contribution table format. For more details, see @SteinmetzNeuro.

contributions of each author, and this identifies the role and value of each team member. More nuanced ways of recording the contributions are in the contribution table using CRediT taxonomy Brand et al., 2015. These newer records provide a better snapshot of each scientist's work in a machinereadable format (Fig. 2). It would be a major step forward if journals would expand the representation of diverse contributions to publications. Biosketches have begun to include a consideration of these contributions; however, it would be useful to provide a clear, standardized method that could also be incorporated into full academic CVs. National Institutes of Health and National Science Foundation now support use of ORCID ID and the National Institutes of Health SciENcv for creating bios. Thus, incorporating a standardized method in those platforms will be critical.

There are a number of additional issues that need to be considered in the recognition of team science. Implicit bias has been shown to disadvantage women and scientists from underrepresented groups in apportioning credit for ideas and work done. For example, several recent studies in eLife, Journal of Clinical Investigation, and the National Academy of Sciences (respectively, Broderick and Casavelle, 2019; Casadevall et al., 2019; Colwell et al., 2020) show that co-first author order is not equitable across sex. Moreover, some of the most fundamental and critical work required for collaborations to be successful, such as managing, motivating, and aligning people, is not always highly valued inside academia, and often is neglected in designating contributions.

In the face of these concerns, there are many additional ways in which academic leaders can implement changes to address the challenges of team science. Some of these changes are likely to be difficult to implement. But together, these immediate and long-term changes will enable us to move beyond vague promises of good will to a culture of reward for team science. Here are recommendations for implementing true team science changes:

- Include collaboration as a specific criterion for hiring, promotion, and tenure decision. Search committees can ask candidates directly about their contributions. Chair letters supporting promotion/tenure should clearly state intellectual contributions in collaborative work. Promotion committees should request comments about collaborative work from letter writers.

- Funding agencies and research institutions can support and reward team science with seed grants and requests for application for collaboration, especially if the proposed work is intrinsically multidisciplinary or of large scale.

- Journals could revise authorship recognition to reflect the contributions of each author.

- Datasets should be assigned a doi independent of the papers in which they first appear, and a culture of citing datasets should be encouraged, so that scientists get career credit for generating these important resources.

- Advisors and ombudspeople need to develop and discuss best practices for dealing with interpersonal issues within a team, including managing expectations, approaches to credit and co-authorship, and communicate the tremendous benefits of collaborative approaches.

While many of these changes may take time to implement, all researchers can consider several ways of adapting to new research approaches immediately. For example, once a large dataset has been generated for one project, consider whether there are creative and useful secondary analyses that can be executed, developed, and written up by someone who was a middle author on the first paper. Many other changes absolutely need the contribution and the vigorous advocacy by academic leaders to improve our recognition of team science. Perhaps the most important change that we recommend is that scientific leadership be aware of, and recognize, the importance of collaboration when conducting evaluations, promotion, and other career decisions. We strongly believe that developing scientists and faculty capable of both leading a research study and contributing to a larger group study are both important contributions to scientific advancement and that these should be recognized and celebrated.

\section{References}

Brand A, Allen L, Altman M, Hlava M, Scott J (2015) Beyond authorship: attribution, contribution, collaboration and credit. Learned Publishin 28:151-155.

Broderick NA, Casadevall A (2019) Gender inequalities among authors who contributed equally. Elife 8:e36399.

Casadevall A, Semenza GL, Jackson S, Tomaselli G, Ahima RS (2019) Reducing bias: accounting for the order of co-first authors. J Clin Invest 129:2167-2168.

Colwell R, Ashley Bear A, Helman A, eds (2020) Promising practices for addressing the underrepresentation of women in science, engineering, and medicine: opening doors. A Consensus Study Report of the National Academies Press. Washington, DC: National Academies. 